\title{
Research on Sports Biomechanics Based on Tennis Serve Technique
}

\author{
Zhiqiang Zhu \\ East China JiaoTong University, Nanchang City, Jiangxi Province, \\ 330013, China
}

\begin{abstract}
In modern society, tennis has not only become one of the most popular sports, but also been included in the Olympic sports, which shows its particularity. Serve technique plays a significant role in tennis sports, which is even a weapon for success. However, according to the actual situation, Chinese athletes' serve technical aspects are significantly worse than foreign athletes'. In this paper, through referring to relevant information, the relevant contents of tennis serve technique are introduced and the sports biomechanics regarding this aspects is studied, which provide a valuable reference for promoting the comprehensive development of tennis in China.

Keywords: tennis; serve technique; sports biomechanics;
\end{abstract}

\section{Toss Stage}

\subsection{Toss position}

Taking toss with right hand for example, in the process of toss, the upper limb joints must be in a straight state, and the position should be in the right front of the body. When toss position is on the right, the ball can be dropped at the highest point, and such position is also conducive to the swing of rocket. The best toss position should be biased in front of the body in order to avoid the influence on the body weight center and rocket location. Only in this way can we ensure that the hit point is just above the body. 
The principle of this toss position is that the difficulty of jump from original position will be far greater than the forward and upward jump, which requires power generating from torso, and then the process of upward rotation of power exertion, that is, body back and side bow. Meanwhile, the pedal and stretch are also needed in order to achieve the purpose of stretching the torso. The effect of vertical movement is one of the best effects in this regard. It is necessary to make the horizontal movement distance effectively controlled to fully achieve this goal. Therefore, all the athletes have their own toss horizontal distance in tennis training[1].

\subsection{Toss fall gap}

Toss fall gap is an important factor that is able to directly affect the quality of service. For many athletes, a consensus has been reached in service that the too high height of the ball throwing does not mean the ball can be served well, which may even lower the quality of service. The reason is that when the ball is thrown to a certain height, its gap with the standard hit point will be greater, and thus changes the speed of the original drop, which will interfere with the prediction of the ball fall position and time, and result in excessive drop error. The player will not be able to hit the ball out. In addition, the too long time of ball fall to the hit point will also have an impact on the athlete's muscle strength, which cannot have a very good control of the ball hit power. It can also be seen the importance of the serve error for the entire serve skill. In order to ensure the quality or to enhance the effectiveness, the drop fall need to be controlled.

\subsection{Toss}

The athletes need to make good preparation when tossing, and make their elbow and ball maintain horizontal. In the process of ball fall, athletes should gradually make elbow straight so that it is able to be close to their ipsilateral thigh. Then straighten the arm from the leg side, and throw the ball. In the whole process, the attention should be paid to the following two points. First, the arm should be straight. Second, the palm should be up. Third, just use the thumb, index finger and middle finger to hold up the ball and fingers should be straight. Fourth, the athletes should avoid the habitual actions such as flicking of wrist and rejection of wrist in the above process. Otherwise, the stability of the ball will not be guaranteed. The athletes will not be able to hit the ball in accordance with their own scheduled route, and even the ball in the air will lose the ability to rotate. Fifth, the best time to drop the ball should be determined. The time usually is at the point that the ball held up to where the arm cannot be up further. If the ball is released, it will go upward freely. If the player throws off the ball too early, the ball will shake in the air and the rotation time increases. When the ball is thrown off too late, the player will lose the initiative to hit the ball, which will affect the ball hit quality[3]. 


\section{Ball Hit Stage}

\subsection{Height of ball hit point}

In the evaluation of the quality of service, the relevant personnel usually considers the height of hit point as the standard. High hit point can increase the chance of ball serve success, namely, the higher the hit point is, the bigger the chance of success will be. Therefore, athletes should hit the ball with all their might. To achieve this effect, athletes need make the lower limb movements in place, forcibly stretch to the top so that the whole body stretches to the fullest, which is the effective way of going up to the highest point for the body gravity center. All of the above actions are require to complete at one time. During the ball hit, the player must control his swing arm to be straight, make a straight line with the racquet, and be perpendicular to the ground. In front view, the front support leg, torso, shoulder, upper limb and racket of the athletes are just a straight line. At the same hitting ball speed, the higher the hit point is, the bigger the probability of ball falling will be in the correct place is, which can enhance the success chance of ball hit. If the drop points are in the same area, the ball speed can be faster. Theoretically, the quality of service is in direct proportion to the height of hit point, which should also be controlled within a certain range[4].

\section{2 "Back-scratching" action stage}

Under normal circumstances, people will use the difference between the height of the racket head lowest point and the center height to reflect the stretch extent of trunk, shoulder and hand. The lower racket head attributes to the bigger stretching of shoulder and abdominal muscles. If the athletes want to make the speed of their upper limbs enhanced, the support of abdominal muscles is needed. When the abdominal muscles work, the power of upper limbs will be instantly enhanced. It can be seen from the analysis of the actual process of the game, for the ordinary athletes, he often deliberately increases the speed in the first episode, and the speed will reduce in the second one. Therefore, it can be derived that in the athletes' cognition, the "back-scratching" action is mainly prepared for the second episode. Only when the speed of first episode is guaranteed, can it ensure that the energy of the abdominal muscles be excited, which is very beneficial for the second episode. In some special games, this situation will be opposite, perhaps because of tactical adjustment or increasing the ball rotation. In this manner, the athletes' "back-scratching" action is more obvious in the second episode[5].

\subsection{Swing and ball hit stage}

When the "back-scratching" is completed, the athletes can prepare for hitting ball and need to adjust the action to hit the ball, which can be divided into two aspects as followings. First, in this process, the athletes need contract the abdominal muscles in the fastest speed, and make the body move forward and upward, so that it can occupy the best position of swing. Second, adjustments should be 
conducted in the grip arm, which is mainly about the shoulder and elbow. Those are important parts of the power output in the process of swing. Therefore, such parts of the active muscles should also be contracted rapidly, which is an important way to improve the speed of swing. When the corresponding action adjustments of the swing and ball hit are completed, the athletes should pay close attention to the direction of the ball, and estimate its trajectory in the air in order to be able to more accurately determine the shot time. When the athletes have decided the time of the hitting, they should hit it immediately. Otherwise, it is very easy to lose the best chance[6].

\subsection{Ball hit action}

Ball hit action must be in line with norms, their physical condition and related hitting habits. At the moment of hitting, the athletes should stretch his body completely and make his gravity center exactly fall on the highest point. At this moment, the athletes' arm should be perpendicular to the racket and the ground, and the ball should be hit at the highest point, which is not easy for many athletes. When the racket comes into contact with the ball, the hand with the racket should respond immediately, namely the power of the wrist is used to drive the arm for internal rotation. This above-mentioned action is the core of the entire action. Therefore, it must not be ignored in the process of ball hit. Otherwise, the ball speed is bound to be affected, and the quality of ball hit will also be lowered. In this case, the scores is difficult to get, and even the ball is hit back immediately. At the same time of the completion of the ball hit action, the athletes' left hand should also act, which should be closed to the chest to maintain the balance of the body to avoid being thrown forward due to the inertia after making too large movement[7].

\section{Describe the Whole Serve Process from the Perspective of Sports Biomechanics}

The tennis serve should be regarded as a coherent entity by analyzing the athletic biology of tennis serve. Serve requires the athletes' own strength, and the source of this strength is not provided by a single part, which requires close coordination of the upper and lower limbs. The legs should bend and stretch. The upper limb should make adjustments to form whip with the torso, rather than simply wave the arm to drop the ball. Just relying on the explosive force not only cannot make the direction of the ball and its speed controlled, but also bring the burden on the athletes' bodies. The correct serve steps are generally as follows. Legs stare, rotate hip, rotate body, forearm rotates around the shoulder, elbow extends, arm spins, turn wrist, act with the ball, and feet touch the ground. If the athletes act well in every step, the quality of each ball serve can be guaranteed. In the process of the serve process division, it can make the following conclusions. First, the station stage. Second, the toss stage. Third, the transitional stage. Fourth, swing 
back stage. Fifth, swing forward and hit ball stage. Six, follow-up stage. Different ways of division are fit for different applicable occasions.

\section{Conclusion}

It is of great significance to study the content of sports biomechanics of tennis serve technique, which is related to not only the development of tennis in China, but also the safety of Chinese athletes during this sports. Serve techniques have always played an important role in tennis, and can sometimes determine the success of the event. Serve is not an independent part. The serve should be considered as a system and should be separately done for different people during training, that is to say, the training should be conducted according to the individual characteristics to enable them to find the most suitable rhythm of the serve. However, it is obvious that this regard is not perfect in China. Therefore, the relevant institutions and personnel should strengthen the study in this aspect.

\section{Acknowledgements}

The research work was supported by Jiangxi Provincial Sports Bureau 2016 research funding, number: 201603.

\section{References}

[1] MU Haibin, WANG Yuxiang. Experimental study on the teaching method of segmented coordination structure in the teaching of tennis serve. Journal of Inner Mongolia Normal University (Natural Science Edition), 01, pp.117-121, 2013.

[2] HU Kai, WANG Yun. Analysis on the serve behavior of elite male tennis players-A case study of serve techniques of Novak Djokovic and Nadal in 2012 Australian Open. Sports Research and Education, 11, pp.104-106, 2013.

[3] HU Kai, WANG Yun. Analysis on the serve quality of tennis serves-Taking Djokovic and Nadal serve technique as an example. Guizhou Sports Science and Technology, 02, pp.43-47, 2013.

[4] SONG ZhiLiang, ZHANG Liang-Xiang. Study on electromyography of excellent male tennis players in flat striking technique. Journal of Jilin Institute of Physical Education, 04, pp.77-81, 2013.

[5] REN Tao, JIANG Hongwei. Analysis on the key points of tennis serve strength training-the stage division based on servetechnique. Journal of Nanjing Physical Education Institute, 06, pp.51-58, 2015.

[6] WANG Huihui, JI Zhongqiu. Study on biomechanics of tennis training effect on tennis serve. Journal of Beijing Normal University (Natural Science Edition), 05, op.658-662, 2016. 
[7] ZHANG Xiyuan, SONG Yawei. Kinematics of tennis movement in the upper part of the link chain whipping action. Journal of Nanjing Institute of Physical Education (Natural Science), 04, pp.22-26, 2015. 